\title{
Use of biosanitizer for removal of carbonates and bicarbonates
}

\author{
Niharika Malviya ${ }^{1}$, Sujata Deo $^{2}$ and Farhin Inam ${ }^{2}$ \\ ${ }^{1}$ Wastewater Technology Division, National Environmental Engineering Research Institute, Nehru Marg, \\ Nagpur-440020, India, ${ }^{2}$ Dept. of Chemistry, Institute of Science, R. T. Road, Civil Lines, Nagpur 440008, India. \\ nihar082003@yahoo.com
}

\begin{abstract}
The physico-chemical anlaysis of groundwater at Kalmeshwar, Nagpur District in India was carried out. The results based on electrical conductivity, total dissolved solids (TDS), chloride and alkalinity revealed that nearly all the water samples were found to be highly polluted. A treatment process comprising the usage of biosanitizer was applied in restoring the polluted groundwater into potable one. By this approach, a reduction of bicarbonate and carbonate content in the ground water was achieved by $90 \%$ and $45 \%$ respectively. Our investigation can be helpful for municipal administrators and in water resource management.
\end{abstract}

Keywords: Biosanitizer, biocatalyst, carbonates, bicarbonates, TDS, water treatment, groundwater, India.

Introduction

The quality of water is of utmost importance. The chemical, physical and bacterial characteristics of groundwater determines its usefulness for municipal, commercial, industrial, agricultural and domestic water usage (Tatawat $\&$ Singh, 2007). Because of continuous growth in population, rapid industrialization and the accompanying technologies involving waste disposals, the rate of discharge of the pollutants into the environment is far exceeding the treatment capacities. The study carried out in the Kalmeshwar industrial and town area is the case study in this direction.

Kalmeshwar is located about $20 \mathrm{~km}$ north-west of Nagpur, a fast developing city in Central India. The Maharashtra Industrial Development Corporation (MIDC) developed an industrial area in Kalmeshwar. The area supports many industries involved in the manufacturing of iron sheets, basic alloys, chemicals, coal products, general engineering equipments and pharmaceuticals (Sharma et al., 2002; Malviya et al., 2008). The study area is of $10 \mathrm{sq} \mathrm{km}$, covered by $1 \mathrm{~m}$ thick fertile black clayey soil. Geologically, the area is underlain by Deccan trap lava of $50-60$ m thickness and 4-7 $\mathrm{m}$ weathered mantle. The water level varies 3- $10 \mathrm{~m}$ below ground level during pre-monsoon and 1.5- $8.5 \mathrm{~m}$ during post-monsoon period. The average hydraulic gradient is $1.5 \mathrm{~m} / \mathrm{km}$ with ground level flow towards north-east direction (Subbarao \& Nawale, 1998; Malviya et al., 2008). The location of sampling stations with respect to distance and direction from the source of pollution is given in (Fig. 1,Table1)

Materials and methods

The monitoring of water quality was carried out for about two and a half years between Aug 2006 to Nov 2008 from eight groundwater sources in Kalmeshwar region of Nagpur District. The sampling sites were selected within $5 \mathrm{~km}$ radius from an iron industry, the source of pollution in the district. Analysis was carried out for assessment of 20 parameters including mineral, demand, nutrient, bacteriological and metal analysis. Parameters including $\mathrm{pH}$, temperature, dissolved oxygen, turbidity and electrical conductivity were monitored on site. Sampling, analysis and preservation of water samples were carried out as per Standard Methods for the Examination of Water and Wastewater (Standard Methods, APHA, AWWA, 2005).

The analyses for all the 20 physico-chemical parameters for 8 samples are given in Table 2. The analysis results were checked for correctness by use of

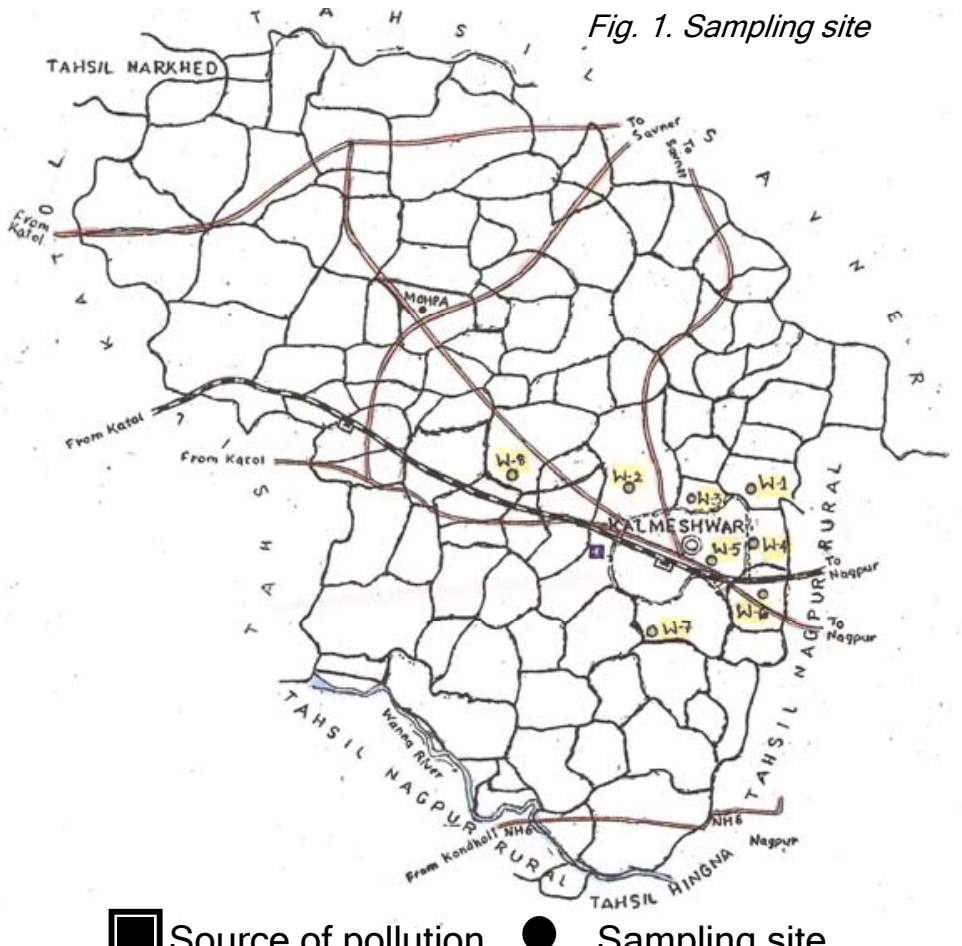

Source of pollution Sampling site

Niharika et al. Indian J.Sci.Technol. 
an automated workbook for checking correctness of water analyses (Bassin, 2007). The respective values for all the 20 parameters were compared with standard limit recommended by Indian Standards for drinking water (Indian Standards IS 10500: 1993).

\section{Biosanitizer}

Biosanitizer (Bhawalkar, 2007; Bhawalkar,\& Bhawalkar, 2008; Malviya et al., 2008) is a catalyst produced from natural ingredients and is a simple tool to manage toxic organics and inorganic converting the waste ingredients into resources. It is available in granular form.

The action is known as 'biosanitization' and controls pathogens too, because pathogens prefer inorganic and toxic organic pollution. Biosanitizer locks the inorganic content of the wastes and cracks the toxic organics to produce safe organics, and also produces active oxygen that takes care of chemical oxygen demand (COD) \& biochemical oxygen demand (BOD), retaining food organics during the treatment.

Biosanitizer features: Biosanitizer is a nano forest. It converts diverse of pollution into resources, at speed that can be 1,000 times higher than the natural ecosystem. No issue of production of sludge or green house gases and no need of machinery and electricity. It is a self operating and self improving system (Bhawalkar, 2007, Bhawalkar,\& Bhawalkar, 2008; Kadbe, 2008).

Biosanitizer applications: It can be put in wells, bore wells, ponds, lakes or in water storage tanks; Produces clean bio-water without pathogens, toxic chemicals and inorganic pollutants; Resists corrosion and contamination;
Vol. 3 No. 2 (Feb 2010)

ISSN: 0974- 6846

Does not allow mosquito breeding and produces high quality farm produce (Kadbe, 2008; Bhawalkar, 2007, Bhawalkar,\& Bhawalkar, 2008).

Biosanitizer dosage is optimized using jar test. Biosanitizer dose in the range of $2.5-15.0 \mathrm{mg}$ per litre with increment of $2.5 \mathrm{mg} / \mathrm{l}$ was tested for optimization. Biosanitizer dosage was directly added to the jars containing one litre carbonate solution of known concentration. After addition of biosanitizer granules, the solution was stirred for $2 \mathrm{~min}$. and left idle for reactor to take place. Biosanitizer granules operate from the bottom of the container and keep on producing oxygen. This drives the natural reactions converting the salty/brackish water to sweet water. The mechanism is similar to that used by the coconut tree in converting saline/brackish water into sweet coconut water. The optimum doze of biosanitizer is found to be 10 $\mathrm{mg} / \mathrm{I}$.

Ten litres each of the eight water samples were used for biosanitizer treatment and the treated water samples were analyzed for different physico-chemical parameters every second day for two months. The analyses result of biosanitizer treatment is shown in Tables 2 (a) - 2 (h) and percent removal efficiency of bicarbonates and carbonates is given in Fig. 2, 3 respectively.

\section{Results and discussions}

The $\mathrm{pH}$ of the water samples is in the range $6.4-7.6$ as presented in Table 2. The TDS concentration of the samples are in the range $491-13530 \mathrm{mg} / \mathrm{l}$ indicating high degree of mineralization. The concentration of $\mathrm{HCO}_{3}, \mathrm{Cl}$, $\mathrm{SO}_{4}$ and $\mathrm{Fe}$ lies in the range of 150-384 mg/l, 15-4655 $\mathrm{mg} / \mathrm{l}, 19-374 \mathrm{mg} / \mathrm{l}$ and $0.12-4.3 \mathrm{mg} / \mathrm{l}$, respectively. Sample

Table 2. Analyses results for samples (W1 to W8)

\begin{tabular}{|c|c|c|c|c|c|c|c|c|}
\hline Parameters & W 1 & W 2 & W 3 & W 4 & W 5 & W 6 & W 7 & W 8 \\
\hline $\mathrm{pH}$ & $6.5-7.3$ & $7.0-7.6$ & $6.6-7.4$ & $6.8-7.3$ & $6.8-7.4$ & $6.9-7.3$ & $6.8-7.5$ & $6.8-7.3$ \\
\hline DO & $0-0.6$ & $1.1-4.0$ & $1.8-3.8$ & $1.2-5.2$ & $0.2-4.2$ & $0-3.6$ & $3.1-4.2$ & $2.6-4.7$ \\
\hline Conductivity & $8390-20500$ & $1160-2520$ & $4500-17050$ & $3900-11230$ & $2070-4350$ & $1865-5170$ & $750-2330$ & $2470-5060$ \\
\hline TDS & $5538-13530$ & $761-1662$ & $3150-11253$ & $2573-7409$ & $1365-2869$ & $1232-3413$ & $491-1634$ & $1731-3336$ \\
\hline COD & $94-360$ & $8-32$ & $172-327$ & 112 - 274 & $32-94$ & $98-214$ & $<8-32$ & $48-146$ \\
\hline BOD & $13-46$ & $<5-8$ & $24-39$ & $16-33$ & $<5-12$ & $14-31$ & $<5-5$ & $7-26$ \\
\hline Alkalinity & $164-380$ & $150-258$ & $195-292$ & $165-236$ & $166-338$ & $250-384$ & $210-332$ & 150 - 252 \\
\hline Hardness & $2180-6200$ & $360-650$ & $1450-5500$ & $1670-3380$ & $840-1500$ & $892-1780$ & $300-1690$ & $880-2600$ \\
\hline $\mathrm{Ca}^{++}$ & $496-1740$ & $79-176$ & $332-1160$ & $332-744$ & $188-299$ & $204-391$ & 77 - 304 & $219-416$ \\
\hline $\mathrm{Mg}^{++}$ & $225-519$ & $7-60$ & $149-630$ & $187-406$ & $84-206$ & $86-250$ & $27-223$ & $38-250$ \\
\hline $\mathrm{Na}^{+}$ & $375-2986$ & $64-393$ & $376-2361$ & $205-1645$ & $163-567$ & $163-725$ & $19-184$ & $210-653$ \\
\hline $\mathrm{K}^{+}$ & $8-61$ & $1-8$ & $8-48$ & $4-33$ & $3-16$ & $3-15$ & $1-6$ & $4-13$ \\
\hline Chloride & $560-4655$ & $56-500$ & $540-3554$ & $290-2548$ & $190-776$ & $140-913$ & $15-50$ & $260-864$ \\
\hline Sulphate & $23-242$ & $35-159$ & $39-206$ & $31-87$ & $49-154$ & $31-374$ & $19-74$ & $75-219$ \\
\hline Phosphate & $0.22-0.42$ & BDL - 0.21 & $0.13-0.22$ & $0.08-0.15$ & BDL - 0.17 & $0.10-0.19$ & $0.11-0.30$ & $0.28-2.6$ \\
\hline Nitrate & $0.21-0.90$ & $0.9-3.5$ & $1.18-2.10$ & $0.9-2.2$ & $1.4-2.6$ & $3.8-9.0$ & $7.1-9.2$ & $2.2-3.6$ \\
\hline Fluoride & $0.11-0.34$ & $0.12-0.31$ & $0.09-0.33$ & BDL - 0.32 & BDL - 0.34 & BDL - 0.35 & BDL - 0.33 & $0.09-0.36$ \\
\hline Iron & $0.81-3.80$ & $0.45-2.2$ & $0.42-2.70$ & $0.26-2.70$ & $0.27-3.10$ & $0.12-2.30$ & $0.44-2.70$ & $0.19-4.30$ \\
\hline Lead & $0.038-0.424$ & BDL - 0.01 & $0.064-0.174$ & $0.068-0.221$ & $0.041-0.123$ & $0.007-0.144$ & BDL - 0.30 & $0.020-0.087$ \\
\hline Coliforms & $>1100$ & $7-21$ & $>1100$ & $7-23$ & $14-43$ & $15-93$ & $9-23$ & $20-240$ \\
\hline
\end{tabular}

Research article

Clndian Society for Education and Environment (iSee)
Biosanitizer

http://www.indjst.org
Niharika et al. Indian J.Sci.Technol. 
W 1 shows the maximum concentration of TDS (5538 $13530 \mathrm{mg} / \mathrm{l})$ and chlorides (560 - $4655 \mathrm{mg} / \mathrm{l})$, while W 7 shows the least concentration of TDS (750 - $2330 \mathrm{mg} / \mathrm{l})$ and chlorides $(15-50 \mathrm{mg} / \mathrm{l})$. Table 3 presents the performance data (average of three sets of biosanitizer treatment). The data clearly indicate that biosanitizer works continuously reducing the alkalinity and hardness thereby reduction in TDS but with marginal increase in chloride and sulphates.

Fig. $2 \& 3$ shows the percent efficiency removal in bicarbonates and carbonates respectively. It can be concluded that the maximum removal efficiency for bicarbonates and carbonates can be achieved within 7 days. The removal efficiency for bicarbonates is around $90 \%$ while for carbonates removal is in the range $40-45 \%$.

Table 3 (b). Analyses results for sample (W2) with use of biosanitizer

\begin{tabular}{|c|c|c|c|c|c|c|c|}
\hline \multirow{3}{*}{ Parameters } & \multicolumn{7}{|c|}{ W-2 } \\
\hline & \multirow{2}{*}{$\begin{array}{c}\text { Values } \\
\text { without } \\
\text { biosanitiz } \\
\text { er }\end{array}$} & \multicolumn{6}{|c|}{ Values with use of biosanitizer } \\
\hline & & $\begin{array}{l}\text { After } \\
1 \text { day }\end{array}$ & $\begin{array}{l}\text { After } \\
3 \text { days }\end{array}$ & $\begin{array}{l}\text { After } \\
5 \text { days }\end{array}$ & $\begin{array}{l}\text { After } \\
7 \text { days }\end{array}$ & $\begin{array}{c}\text { After } \\
15 \text { days }\end{array}$ & $\begin{array}{c}\text { After } \\
1 \text { Month }\end{array}$ \\
\hline $\mathrm{pH}$ & 7.0 & 7.0 & 7.0 & 7.0 & 7.0 & 7.0 & 7.0 \\
\hline Conductivity & 1490 & 1440 & 1350 & 1330 & 1280 & 1240 & 1235 \\
\hline TDS & 1000 & 1038 & 930 & 904 & 871 & 846 & 840 \\
\hline Alkalinity as $\mathrm{HCO}_{3}$ & 268 & 224 & 164 & 120 & 64 & 59 & 55 \\
\hline Total hardness as $\mathrm{CO}_{3}$ & 534 & 506 & 410 & 364 & 312 & 309 & 305 \\
\hline Calcium as $\mathrm{CaCO}_{3}$ & 410 & 374 & 328 & 299 & 287 & 247 & 238 \\
\hline Magnesium as $\mathrm{MgCO}_{3}$ & 124 & 132 & 82 & 65 & 25 & 62 & 67 \\
\hline $\mathrm{Ca}^{++}$ & 164 & 149 & 131 & 120 & 115 & 99 & 95 \\
\hline $\mathrm{Mg}^{++}$ & 30 & 32 & 19 & 16 & 6 & 15 & 16 \\
\hline $\mathrm{Na}^{+}$ & 179 & 206 & 198 & 203 & 204 & 197 & 199 \\
\hline $\mathrm{K}^{+}$ & 4 & 4 & 4 & 4 & 9 & 6 & 8 \\
\hline Chloride & 220 & 265 & 250 & 254 & 261 & 260 & 264 \\
\hline Sulphate & 76 & 79 & 84 & 87 & 89 & 79 & 76 \\
\hline
\end{tabular}

All parameter are in $\mathrm{mg} / \mathrm{l}$ accept for $\mathrm{pH}$, conductivity and coli forms; unit for conductivity is $\mu \mathrm{S} / \mathrm{cm}$

\begin{tabular}{|c|c|c|c|c|c|c|c|}
\hline \multirow{3}{*}{ Parameters } & \multicolumn{7}{|c|}{ W-3 } \\
\hline & \multirow{2}{*}{$\begin{array}{c}\text { Values } \\
\text { without } \\
\text { biosanitizer }\end{array}$} & \multicolumn{6}{|c|}{ Values with use of biosanitizer } \\
\hline & & $\begin{array}{l}\text { After } \\
1 \text { day }\end{array}$ & $\begin{array}{c}\text { After } \\
3 \text { days }\end{array}$ & $\begin{array}{c}\text { After } \\
5 \text { days }\end{array}$ & $\begin{array}{l}\text { After } \\
7 \text { days }\end{array}$ & $\begin{array}{c}\text { After } \\
15 \text { days }\end{array}$ & $\begin{array}{c}\text { After } \\
1 \text { Month }\end{array}$ \\
\hline $\mathrm{pH}$ & 7.2 & 7.0 & 7.0 & 7.0 & 7.0 & 7.0 & 7.0 \\
\hline Conductivity & 8495 & 8255 & 8160 & 7900 & 7760 & 7910 & 7970 \\
\hline TDS & 5777 & 5698 & 5549 & 5365 & 5311 & 5380 & 5418 \\
\hline Alkalinity as $\mathrm{HCO}_{3}$ & 292 & 144 & 88 & 64 & 47 & 44 & 40 \\
\hline Total hardness as $\mathrm{CO}_{3}$ & 2680 & 2560 & 2400 & 2180 & 2050 & 2035 & 2010 \\
\hline Calcium as $\mathrm{CaCO}_{3}$ & 1740 & 1530 & 1515 & 1310 & 1180 & 1245 & 1195 \\
\hline Magnesium as $\mathrm{MgCO}_{3}$ & 940 & 1030 & 885 & 870 & 870 & 790 & 815 \\
\hline $\mathrm{Ca}^{++}$ & 696 & 612 & 606 & 524 & 472 & 488 & 478 \\
\hline $\mathrm{Mg}^{++}$ & 225 & 247 & 212 & 209 & 209 & 190 & 196 \\
\hline $\mathrm{Na}^{+}$ & 1253 & 1248 & 1244 & 1255 & 1285 & 1321 & 1334 \\
\hline $\mathrm{K}^{+}$ & 25 & 38 & 38 & 39 & 40 & 41 & 44 \\
\hline Chloride & 1865 & 1873 & 1868 & 1882 & 1931 & 1983 & 2011 \\
\hline Sulphate & 137 & 144 & 142 & 148 & 145 & 151 & 148 \\
\hline
\end{tabular}

All parameter are in $\mathrm{mg} / \mathrm{l}$ accept for $\mathrm{pH}$, conductivity and coli forms; unit for conductivity is $\mu \mathrm{S} / \mathrm{cm}$

\begin{tabular}{|c|c|c|c|c|c|c|c|}
\hline \multirow{3}{*}{ Parameters } & \multicolumn{7}{|c|}{$W-1$} \\
\hline & \multirow{2}{*}{$\begin{array}{c}\text { Values } \\
\text { without } \\
\text { biosanitizer }\end{array}$} & \multicolumn{6}{|c|}{ Values with use of biosanitizer } \\
\hline & & $\begin{array}{l}\text { After } \\
1 \text { day }\end{array}$ & $\begin{array}{c}\text { After } \\
3 \text { days }\end{array}$ & $\begin{array}{c}\text { After } \\
5 \text { days }\end{array}$ & $\begin{array}{c}\text { After } \\
7 \text { days }\end{array}$ & $\begin{array}{c}\text { After } \\
15 \text { days }\end{array}$ & $\begin{array}{c}\text { After } \\
1 \text { Month }\end{array}$ \\
\hline $\mathrm{pH}$ & 7.1 & 7.1 & 7.1 & 7.0 & 7.0 & 7.0 & 7.0 \\
\hline Conductivity & 13910 & 13810 & 13330 & 12875 & 12450 & 12225 & 12050 \\
\hline TDS & 9320 & 9254 & 9064 & 8736 & 8468 & 8435 & 8437 \\
\hline Alkalinity as $\mathrm{HCO}_{3}$ & 286 & 140 & 64 & 56 & 36 & 32 & 30 \\
\hline Total hardness as $\mathrm{CO}_{3}$ & 5200 & 5080 & 4840 & 4420 & 4110 & 4066 & 4025 \\
\hline Calcium as $\mathrm{CaCO}_{3}$ & 4000 & 3803 & 3540 & 3540 & 2960 & 2887 & 2857 \\
\hline Magnesium as $\mathrm{MgCO}_{3}$ & 1200 & 1277 & 1300 & 880 & 1150 & 1197 & 1168 \\
\hline $\mathrm{Ca}^{++}$ & 1600 & 1521 & 1416 & 1416 & 1184 & 1155 & 1143 \\
\hline $\mathrm{Mg}^{++}$ & 288 & 306 & 312 & 211 & 276 & 283 & 280 \\
\hline $\mathrm{Na}^{+}$ & 1647 & 1672 & 1691 & 1700 & 1733 & 1736 & 1754 \\
\hline $\mathrm{K}^{+}$ & 42 & 43 & 43 & 44 & 44 & 47 & 45 \\
\hline Chloride & 2528 & 2564 & 2581 & 2601 & 2647 & 2654 & 2681 \\
\hline Sulphate & 95 & 102 & 117 & 113 & 118 & 121 & 120 \\
\hline
\end{tabular}

All parameter are in $\mathrm{mg} / \mathrm{l}$ accept for $\mathrm{pH}$, conductivity and coli forms; unit for conductivity is $\mu \mathrm{S} / \mathrm{cm}$

\section{Conclusion}

The groundwater in Kalmeshwar town area was highly polluted as the TDS $(2000 \mathrm{mg} / \mathrm{L})$, total carbonates $(600 \mathrm{mg} / \mathrm{L})$, chlorides $(1000 \mathrm{mg} / \mathrm{L})$, calcium (200 $\mathrm{mg} / \mathrm{L})$, magnesium $(100 \mathrm{mg} / \mathrm{L})$ and iron $(1.0 \mathrm{mg} / \mathrm{L})$ have crossed the maximum permissible limits of ISO for drinking water (Indian Standards IS 10500 : 1993). Application of biosanitizer has improved the quality of water by reducing the values of bicarbonates and carbonates due to which the washing ability of water has increased. Along with reduction in bicarbonates and carbonates there is marginal increase in chlorides and sulphates. As the values of increased parameters are well within the permissible limits except for W-1, W- 3 and W4 this increase in chlorides and sulphates can be neglected. The maximum removal efficiency for bicarbonates and carbonates can be achieved within 7 days. The removal efficiency for bicarbonates is around $90 \%$ while for carbonates removal is in the range 40 - 45 percent. Water can be treated to safe levels through use of biosanitizer. Thus, the biosanitizer can be one of the solutions for the next generation in water treatment. Studies for determining the reason for increase in chlorides and sulphates are in progress.

\section{Acknowledgement}

The authors acknowledge the help of Mr. Arvind B. Kadbe in inspiring the study and providing biosanitizer. The work was done in Institute of Science, Nagpur as part of
Research article

Clndian Society for Education and Environment (iSee)
Biosanitizer

http://www.indjst.org
Niharika et al. Indian J.Sci.Technol. 
Table 3 (d). Analyses results for sample (W4) with use of biosanitizer

\begin{tabular}{|c|c|c|c|c|c|c|c|}
\hline \multirow{3}{*}{ Parameters } & \multicolumn{7}{|c|}{ W-4 } \\
\hline & \multirow{2}{*}{$\begin{array}{c}\text { Values } \\
\text { without } \\
\text { biosanitizer }\end{array}$} & \multicolumn{6}{|c|}{ Values with use of biosanitizer } \\
\hline & & $\begin{array}{l}\text { After } \\
1 \text { day }\end{array}$ & $\begin{array}{c}\text { After } \\
3 \text { days }\end{array}$ & $\begin{array}{c}\text { After } \\
5 \text { days }\end{array}$ & $\begin{array}{c}\text { After } \\
7 \text { days }\end{array}$ & $\begin{array}{c}\text { After } \\
15 \text { days }\end{array}$ & $\begin{array}{c}\text { After } \\
1 \text { Month }\end{array}$ \\
\hline $\mathrm{pH}$ & 7.0 & 7.0 & 7.0 & 7.0 & 6.9 & 6.9 & 6.9 \\
\hline Conductivity & 8140 & 8190 & 7840 & 7575 & 7430 & 7565 & 7510 \\
\hline TDS & 5455 & 5567 & 5255 & 5151 & 5038 & 5143 & 5122 \\
\hline Alkalinity as $\mathrm{HCO}_{3}$ & 164 & 147 & 123 & 98 & 58 & 49 & 46 \\
\hline Total hardness as $\mathrm{CO}_{3}$ & 2380 & 2350 & 2040 & 1695 & 1510 & 1470 & 1405 \\
\hline Calcium as $\mathrm{CaCO}_{3}$ & 1710 & 1410 & 1345 & 1280 & 1060 & 1075 & 1065 \\
\hline Magnesium as $\mathrm{MgCO}_{3}$ & 670 & 940 & 695 & 415 & 450 & 395 & 340 \\
\hline $\mathrm{Ca}^{++}$ & 684 & 564 & 538 & 512 & 424 & 430 & 426 \\
\hline $\mathrm{Mg}^{++}$ & 161 & 226 & 167 & 97 & 108 & 95 & 82 \\
\hline $\mathrm{Na}^{+}$ & 1223 & 1287 & 1272 & 1350 & 1375 & 1428 & 1438 \\
\hline $\mathrm{K}^{+}$ & 25 & 33 & 32 & 35 & 35 & 37 & 40 \\
\hline Chloride & 1882 & 1990 & 1965 & 2090 & 2118 & 2204 & 2227 \\
\hline Sulphate & 52 & 56 & 57 & 57 & 71 & 68 & 65 \\
\hline
\end{tabular}

All parameter are in $\mathrm{mg} / \mathrm{l}$ accept for $\mathrm{pH}$, conductivity and coli forms; unit for conductivity is $\mu \mathrm{S} / \mathrm{cm}$

\begin{tabular}{|c|c|c|c|c|c|c|c|}
\hline \multirow{3}{*}{ Parameters } & \multicolumn{7}{|c|}{ W-7 } \\
\hline & \multirow{2}{*}{$\begin{array}{c}\text { Values } \\
\text { without } \\
\text { biosanitizer }\end{array}$} & \multicolumn{6}{|c|}{ Values with use of biosanitizer } \\
\hline & & $\begin{array}{l}\text { After } \\
1 \text { day }\end{array}$ & $\begin{array}{c}\text { After } \\
3 \text { days }\end{array}$ & $\begin{array}{c}\text { After } \\
5 \text { days }\end{array}$ & $\begin{array}{c}\text { After } \\
7 \text { days }\end{array}$ & $\begin{array}{c}\text { After } \\
15 \text { days }\end{array}$ & $\begin{array}{c}\text { After } \\
1 \text { Month }\end{array}$ \\
\hline $\mathrm{pH}$ & 7.1 & 7.1 & 7.1 & 7.0 & 7.0 & 7.0 & 7.0 \\
\hline Conductivity & 920 & 870 & 760 & 530 & 450 & 450 & 435 \\
\hline TDS & 624 & 591 & 508 & 348 & 297 & 298 & 286 \\
\hline Alkalinity as $\mathrm{HCO}_{3}$ & 264 & 246 & 210 & 132 & 102 & 94 & 89 \\
\hline Total hardness as $\mathrm{CO}_{3}$ & 312 & 276 & 256 & 224 & 194 & 192 & 189 \\
\hline Calcium as $\mathrm{CaCO}_{3}$ & 174 & 144 & 100 & 71 & 78 & 76 & 79 \\
\hline Magnesium as $\mathrm{MgCO}_{3}$ & 138 & 132 & 156 & 153 & 116 & 116 & 110 \\
\hline $\mathrm{Ca}^{++}$ & 70 & 58 & 40 & 28 & 31 & 30 & 32 \\
\hline $\mathrm{Mg}^{++}$ & 33 & 32 & 37 & 37 & 29 & 28 & 26 \\
\hline $\mathrm{Na}^{+}$ & 134 & 133 & 110 & 55 & 42 & 43 & 38 \\
\hline $\mathrm{K}^{+}$ & 3 & 4 & 3 & 2 & 2 & 1 & 2 \\
\hline Chloride & 27 & 26 & 27 & 28 & 28 & 33 & 30 \\
\hline Sulphate & 35 & 38 & 35 & 37 & 42 & 48 & 45 \\
\hline
\end{tabular}

All parameter are in $\mathrm{mg} / \mathrm{l}$ accept for $\mathrm{pH}$, conductivity and coli forms; unit for conductivity is $\mu \mathrm{S} / \mathrm{cm}$

\begin{tabular}{|c|c|c|c|c|c|c|c|}
\hline \multirow{3}{*}{ Parameters } & \multicolumn{7}{|c|}{ W-5 } \\
\hline & \multirow{2}{*}{$\begin{array}{c}\text { Values } \\
\text { without } \\
\text { biosanitizer }\end{array}$} & \multicolumn{6}{|c|}{ Values with use of biosanitizer } \\
\hline & & $\begin{array}{l}\text { After } \\
1 \text { day }\end{array}$ & $\begin{array}{c}\text { After } \\
3 \text { days }\end{array}$ & $\begin{array}{c}\text { After } \\
5 \text { days }\end{array}$ & $\begin{array}{c}\text { After } \\
7 \text { days }\end{array}$ & $\begin{array}{c}\text { After } \\
15 \text { days }\end{array}$ & $\begin{array}{c}\text { After } \\
1 \text { Month }\end{array}$ \\
\hline $\mathrm{pH}$ & 7.0 & 7.0 & 7.0 & 7.0 & 7.0 & 6.9 & 6.9 \\
\hline Conductivity & 2925 & 2980 & 2905 & 2805 & 2800 & 2670 & 2620 \\
\hline TDS & 1990 & 2024 & 1978 & 1916 & 1921 & 1787 & 1778 \\
\hline Alkalinity as $\mathrm{HCO}_{3}$ & 322 & 302 & 226 & 134 & 78 & 73 & 80 \\
\hline Total hardness as $\mathrm{CO}_{3}$ & 760 & 730 & 645 & 587 & 510 & 500 & 490 \\
\hline Calcium as $\mathrm{CaCO}_{3}$ & 552 & 533 & 454 & 432 & 357 & 370 & 358 \\
\hline Magnesium as $\mathrm{MgCO}_{3}$ & 208 & 197 & 191 & 164 & 153 & 130 & 132 \\
\hline $\mathrm{Ca}^{++}$ & 221 & 213 & 181 & 169 & 143 & 148 & 143 \\
\hline $\mathrm{Mg}^{++}$ & 50 & 72 & 46 & 39 & 37 & 31 & 32 \\
\hline $\mathrm{Na}^{+}$ & 480 & 505 & 518 & 516 & 541 & 497 & 495 \\
\hline $\mathrm{K}^{+}$ & 10 & 13 & 11 & 10 & 14 & 10 & 12 \\
\hline Chloride & 700 & 736 & 751 & 745 & 784 & 725 & 721 \\
\hline Sulphate & 73 & 77 & 85 & 87 & 93 & 75 & 81 \\
\hline
\end{tabular}

All parameter are in $\mathrm{mg} / \mathrm{l}$ accept for $\mathrm{pH}$, conductivity and coli forms; unit for conductivity is $\mu \mathrm{S} / \mathrm{cm}$ Ph.D. course.

\section{References}

1. Bassin JK (2007) An automated workbook for checking correctness of water analyses. J. Indian Water Works Asso. 39, 259-264.
2. Bhawalkar US (2007) Sanitation in human habitation. Indian Plumbing Today. 4, 2631.

3. Bhawalkar US and Bhawalkar SU (2008) Invisible compact and high rate phytoremediation of water an wastewater using biosanitizer ecotechnology. $11^{\text {th }}$ Intl. Conf. on Wetland Systems Technol. in Water Pollution Control, Nov. 1-7, Indore.

4. CSE (2005) A wastewater recycling manual for urban areas, with case studies. Publ. by Centre for Sci. \& Environ., New Delhi.

5. Indian Standards : Drinking Water - Specifications (IS 10500 : 1993).

6. Kadbe Arvind (2008) Turning pollution to profit - biosanitizer. The Hitavada. $6^{\text {th }} \& 13^{\text {th }}$ January.

7. Malviya NM, Deo $S$ and Inam $F$ (2008) Reduction in alkalinity and hardness by the use of biosanitizer at Kalmeshwar in Nagpur District. Intl. Conf. on Water Crisis- Challenges \& Opportunities, 28- 9 Feb., Nagpur.

8. Sharma SK, Tiwari AN and Nawale VP (2002) Impact of Industrial Pollution on Groundwater Quality in Kalmeshwar Area, Nagpur District, Maharashtra", Proceedings of National Conference on Pollution Prevention and Control in India: IAEM, pp 183-188.

9. Standard Methods for the Examination of Water and Wastewater, $21^{\text {st }}$ Edition 2005, APHA, AWWA.

10. Subbarao D and Nawale V.P., 1998, Report on Ground Water Pollution in Kalmeshwar area, Nagpur district, Maharashtra, CGWB, CR, Nagpur.

11. Tatawat Rakesh Kumar and Singh Chandel CP (2007) Quality of groundwater of Jaipur-City, Rajasthan, (India) and its suitability for domestic and irrigation purpose. Appl. Ecol. Environ. Res., 6 (2), 79-88.
Research article

CIndian Society for Education and Environment (iSee)
Niharika et al. Indian J.Sci.Technol. 
Table 3 (f). Analyses results for sample (W6) with use of biosanitizer

\begin{tabular}{|c|c|c|c|c|c|c|c|}
\hline \multirow{3}{*}{ Parameters } & \multicolumn{7}{|c|}{ W-6 } \\
\hline & \multirow{2}{*}{$\begin{array}{c}\text { Values } \\
\text { without } \\
\text { biosanitizer }\end{array}$} & \multicolumn{6}{|c|}{ Values with use of biosanitizer } \\
\hline & & $\begin{array}{l}\text { After } \\
1 \text { day }\end{array}$ & $\begin{array}{c}\text { After } \\
3 \text { days }\end{array}$ & $\begin{array}{c}\text { After } \\
5 \text { days }\end{array}$ & $\begin{array}{c}\text { After } \\
7 \text { days }\end{array}$ & $\begin{array}{c}\text { After } \\
15 \text { days }\end{array}$ & $\begin{array}{c}\text { After } \\
1 \text { Month }\end{array}$ \\
\hline $\mathrm{pH}$ & 7.0 & 7.0 & 7.0 & 7.0 & 7.0 & 7.0 & 7.0 \\
\hline Conductivity & 4015 & 3855 & 3780 & 3770 & 3775 & 3835 & 3825 \\
\hline TDS & 2732 & 2622 & 2570 & 2583 & 2596 & 2618 & 2600 \\
\hline Alkalinity as $\mathrm{HCO}_{3}$ & 340 & 328 & 270 & 174 & 92 & 88 & 85 \\
\hline Total hardness as $\mathrm{CO}_{3}$ & 964 & 832 & 728 & 651 & 595 & 588 & 585 \\
\hline Calcium as $\mathrm{CaCO}_{3}$ & 486 & 476 & 408 & 338 & 298 & 335 & 328 \\
\hline Magnesium as $\mathrm{MgCO}_{3}$ & 478 & 356 & 320 & 313 & 297 & 253 & 257 \\
\hline $\mathrm{Ca}^{++}$ & 194 & 190 & 163 & 135 & 119 & 134 & 131 \\
\hline $\mathrm{Mg}^{++}$ & 115 & 85 & 77 & 75 & 71 & 61 & 62 \\
\hline $\mathrm{Na}^{+}$ & 683 & 684 & 701 & 736 & 758 & 765 & 757 \\
\hline $\mathrm{K}^{+}$ & 14 & 17 & 15 & 15 & 19 & 20 & 22 \\
\hline Chloride & 860 & 882 & 890 & 941 & 976 & 984 & 981 \\
\hline Sulphate & 288 & 267 & 289 & 292 & 298 & 302 & 296 \\
\hline
\end{tabular}

All parameter are in $\mathrm{mg} / \mathrm{l}$ accept for $\mathrm{pH}$, conductivity and coli forms; unit for conductivity is $\mu \mathrm{S} / \mathrm{cm}$

Table 3 (h). Analyses results for sample (W8) with use of biosanitizer

\begin{tabular}{|c|c|c|c|c|c|c|c|}
\hline \multirow{3}{*}{ Parameters } & \multicolumn{7}{|c|}{ W-8 } \\
\hline & \multirow{2}{*}{$\begin{array}{l}\text { Values without } \\
\text { biosanitizer }\end{array}$} & \multicolumn{6}{|c|}{ Values with use of biosanitizer } \\
\hline & & $\begin{array}{l}\text { After } \\
1 \text { day }\end{array}$ & $\begin{array}{c}\text { After } \\
3 \text { days }\end{array}$ & $\begin{array}{c}\text { After } \\
5 \text { days }\end{array}$ & $\begin{array}{l}\text { After } \\
7 \text { days }\end{array}$ & $\begin{array}{c}\text { After } \\
15 \text { days }\end{array}$ & $\begin{array}{c}\text { After } \\
1 \text { Month }\end{array}$ \\
\hline $\mathrm{pH}$ & 7.1 & 7.1 & 7.1 & 7.0 & 7.0 & 7.0 & 7.0 \\
\hline Conductivity & 3060 & 3090 & 2960 & 2840 & 2860 & 2855 & 2880 \\
\hline TDS & 2081 & 2131 & 2014 & 1923 & 1975 & 1914 & 1932 \\
\hline Alkalinity as $\mathrm{HCO}_{3}$ & 270 & 196 & 116 & 88 & 72 & 68 & 65 \\
\hline Total hardness as $\mathrm{CO}_{3}$ & 1092 & 1020 & 952 & 800 & 762 & 753 & 748 \\
\hline Calcium as $\mathrm{CaCO}_{3}$ & 752 & 670 & 596 & 520 & 497 & 512 & 508 \\
\hline Magnesium as $\mathrm{MgCO}_{3}$ & 340 & 350 & 356 & 280 & 265 & 241 & 240 \\
\hline $\mathrm{Ca}^{++}$ & 301 & 268 & 238 & 208 & 199 & 205 & 203 \\
\hline $\mathrm{Mg}^{++}$ & 82 & 84 & 85 & 67 & 63 & 58 & 57 \\
\hline $\mathrm{Na}^{+}$ & 386 & 434 & 417 & 435 & 469 & 447 & 455 \\
\hline $\mathrm{K}^{+}$ & 10 & 11 & 11 & 11 & 13 & 11 & 13 \\
\hline Chloride & 510 & 580 & 555 & 585 & 641 & 600 & 616 \\
\hline Sulphate & 136 & 142 & 139 & 138 & 135 & 141 & 142 \\
\hline
\end{tabular}

All parameter are in mg/l accept for $\mathrm{pH}$, conductivity and coli forms; unit for conductivity is $\mu \mathrm{S} / \mathrm{cm}$

Niharika et al. Indian J.Sci.Technol. 


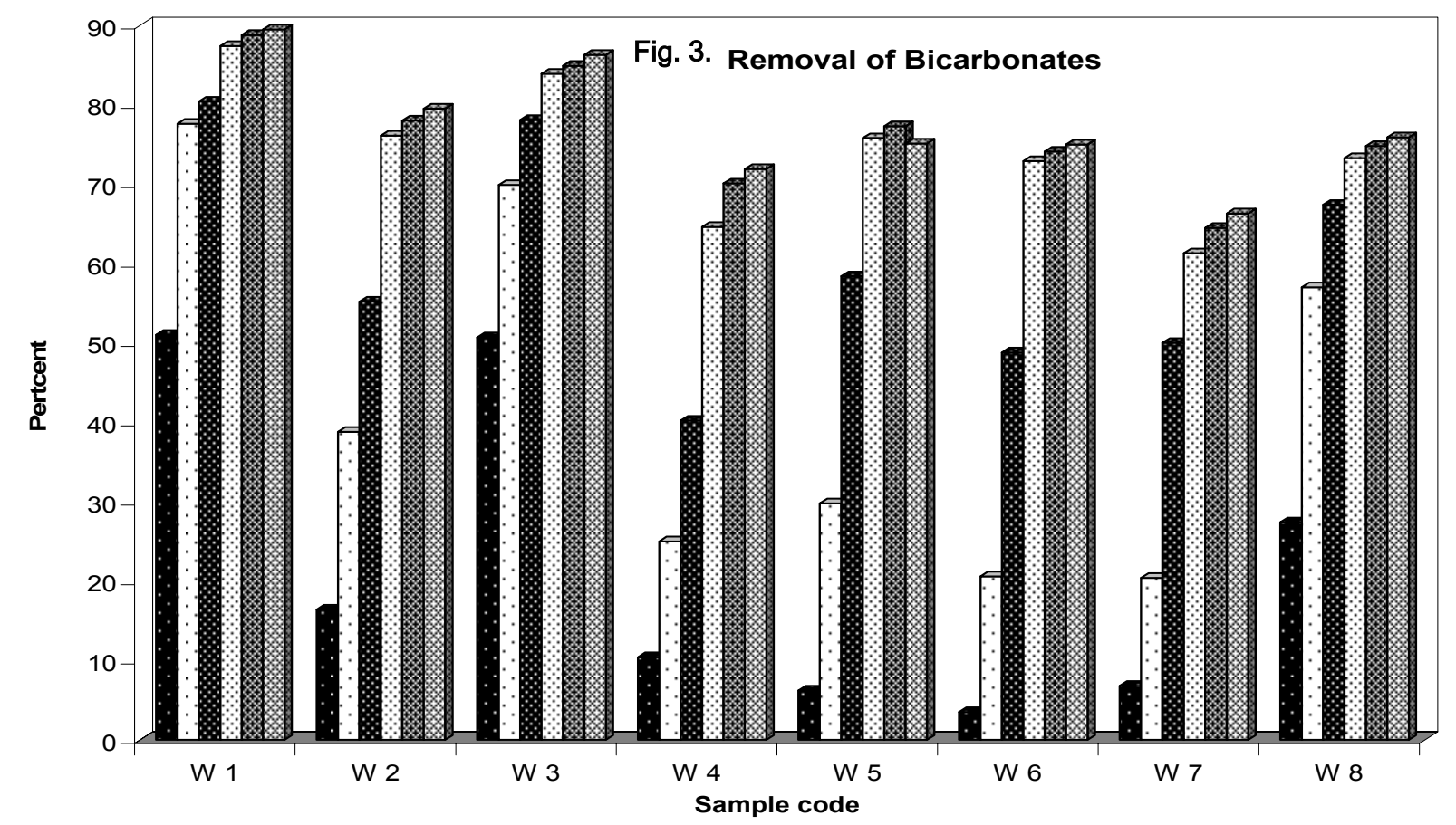

- After 1 day use $\square$ After 3 days use After 5 days use $\mathbf{0}$ After 7 days use After 15 days use $\quad$ After 30 days use

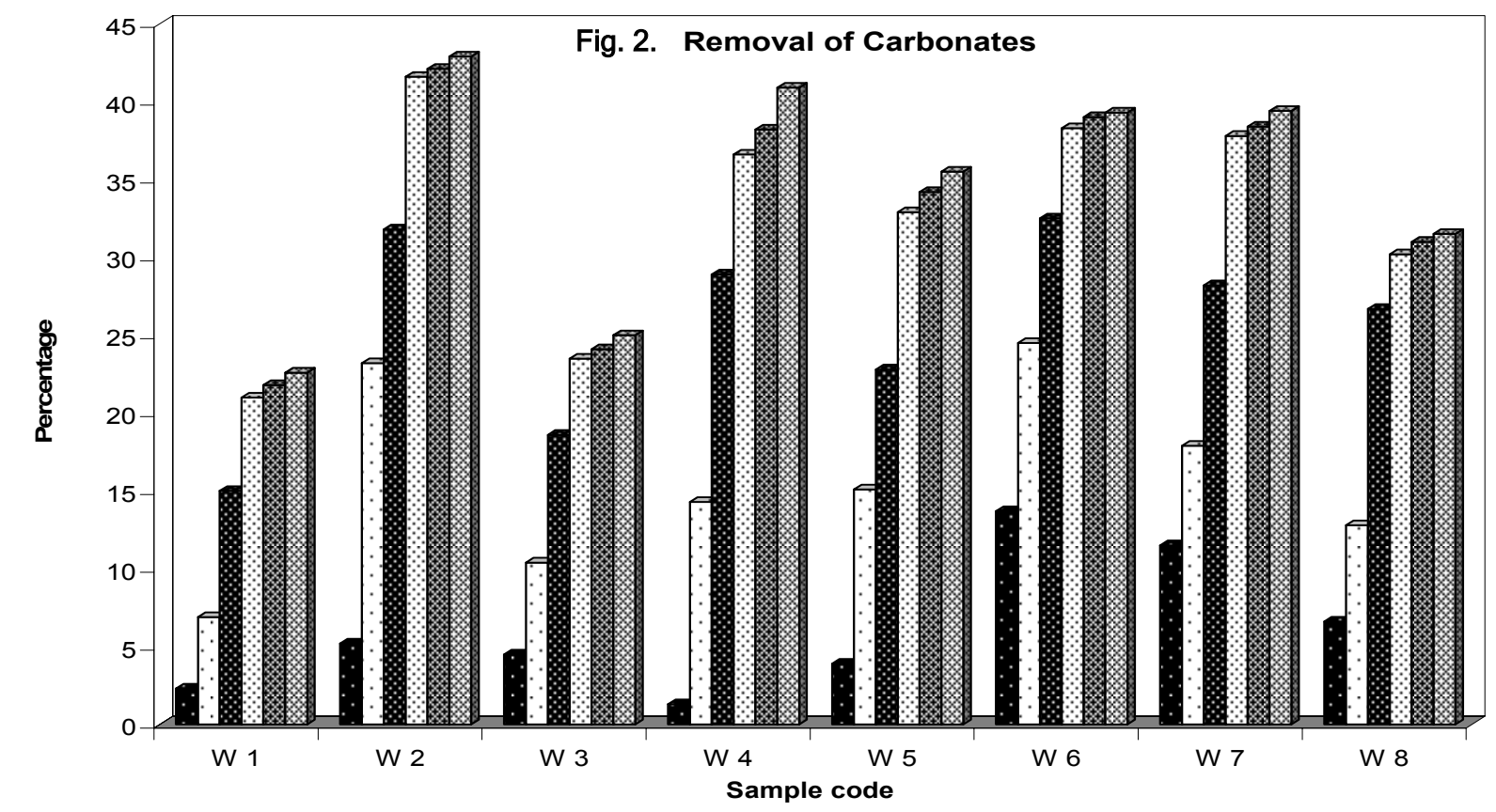

After 1 day use $\square$ After 3 days use After 5 days use

After 7 days use

After 15 days use After 30 days use 